\title{
The Price is Right: Predicting Reagent Prices
}

\author{
Kwabena Ofori-Atta, Clayton Springer*
}

The Novartis Institute for BioMedical Research.

Reagent Pricing. Machine learning. Random forest. Cliff pairs, Matched molecular pairs.

\begin{abstract}
We present a model for estimating the price of a reagent from its chemical structure. It is intended to be useful when doing reagent selection for library design. The model is a Random Forest regressor which is trained on the MolPort catalog of 302K reagents and the log of their price. For descriptors we use topological fingerprints from RDKit: chiral Morgan fingerprints, its medicinal chemistry descriptors, and counts of undetermined chiral centers. The model has an out-of-bag performance of $34 \%$ variance explained in $\log$ Price. When predicting on known reagents, the model explains $91 \%$ of the variance in $\log$ Price. We analyzed the model to understand the errors that the model makes. We show that the compounds with the highest errors have only a subtly different structure from similar molecules, but very different in price.
\end{abstract}

\section{Introduction}

2 Chemical libraries typically decorate an advanced intermediate with reagents from a set of commercially available reagents. The 3 process of designing a chemical library involves maximizing a library's potency, diversity, and/or ADME properties [1] and there 4 are a variety of approaches for doing that. The design can be constructed by the final products or reagents via cheminformatics, 5 pharmacophoric, or structure based approaches [1-14].

6 Like potency, diversity, and ADME properties, the cost of the chemical reagents used in a library is an important consideration 7 in what compounds are actually made. In principle, the price of a reagent should just be a simple lookup. However, it is our experi8 ence that the price information is often missing from reagent lists, and a model that could estimate the price for a chemical com9 pound would be a great convenience. There are analyses of prices of reagents [15], estimates of synthetic accessibility [16], and one 
published model for estimating the expense of almost 4000 compounds used for biological assays [17]. This work focuses on the

11 estimating the cost of a commercial reagent. This would be important in the context of buying a set of reagents for a chemical reac-

tion step. Also we present an approach to investigate errors in the model.

Including an estimated price in the design will allow us to avoid one cause of library changes after reagent selection finalization and library construction. Since libraries are designed as a collective set of compounds, last-minute reagent substitutions can undermine the coverage of the library, particularly in cases where a limited number of compounds were selected as a designed set to address specific scientific questions or to remove statistical bias-for example, following the principles of statistical experimental design.

\section{Introduction}

An economical way to generate a chemical libraries is to combine a proprietary, highly engineered reagent with a commercially available reagent. Chemical libraries can be designed to maximize potency, diversity, and/or ADME properties [1]. One can take various design approaches including analyzing the final products or reagents via cheminformatics, pharmacophoric, or structure based approaches [1-14]. However, if the commercial reagents selected are too expensive then the designed library would no longer be economical. While there are many publications on various aspects of library design, there relatively few that address the issue of cost: there are analyses of prices of reagents [15], estimates of synthetic accessibility [16], and one published model for estimating the expense of almost 4,000 compounds used for biological assays [17].

Including an estimated price in the design will allow us to avoid one cause of library changes after reagent selection finalization and library construction. Since libraries are designed as a collective set of compounds, last-minute reagent substitutions can undermine the coverage of the library, particularly in cases where a limited number of compounds were selected as a designed set to address specific scientific questions or to remove statistical bias-for example, following the principles of statistical experimental design.

31 In principle, the price of a reagent should just be a simple lookup. However, it is our experience that the price information is often missing from reagent lists, and a model that could estimate the price for a chemical compound would be a great convenience. In the context of buying a set of reagents for a single chemical reaction step, the total of the cost of reagents is a key property of the library. This work focuses on the estimating the cost of each commercial reagent.

\section{Methods}

\section{Data source - price transformation}

We treat the price prediction model effort as a machine learning problem. The analysis began with obtaining and processing the 
Within the file, each reagent was presented in SMILES notation and listed with an associated price range that matches one of five distinct price categories (see Table 1). For purposes of this analysis, we also assigned a single numerical value for each price range.

41 A price range was given for $1 \mathrm{mg}, 5 \mathrm{mg}$, and 50mg amounts of each reagent. To prepare the data, reagents within the catalog were converted from the SMILES format to RDKit molecular objects. The $\log _{10}$ of the numerical value for each price range for the $50 \mathrm{mg}$ size was used as the training set in this study.

\section{Descriptors}

To analyze chemicals via Random Forest regression, the reagents were translated into a vector of descriptors. We used the RDKit to calculate the descriptors of each reagent: the counts of the hashed chiral Morgan fingerprints (hashed into: 8,192 bins; radius: 3), the counts of the number of undetermined and determined chiral centers, and RDKit's Descriptor set [18]. Ultimately, there were 8,385 elements in the final descriptor vector of each reagent.

\section{ML technique}

The machine learning model was created from scikit-learn's Random Forest regressor library (scikit-learn version 0.17) [19, 20].

51 The random forest model was composed of 1,000 decision trees. The settings for each random forest construction are: 'bootstrap': True, 'criterion': 'mse', 'max_depth': None, 'max_features': 'auto', 'max_leaf_nodes': None, 'min_samples_leaf': 1, 'min_samples_split': 2, 'min_weight_fraction_leaf': 0.0,'n_jobs': 1, 'oob_score': True, 'random_state': None, 'verbose': 0, 'warm_start': False.

54 For the out-of-bag (OOB) samples of each tree we used the default setting of a random selection. This leads to a fraction of $\left(1-\frac{1}{e}\right)$ $\sim 37 \%$ held out in each bag which is approximately 112,094 examples held out in each bag.

\section{Model performance}

We have two training/test data set splits on which we calculate our metrics of model performance: 'out-of-bag' (OOB) and 'in catalog' (Table 2). The OOB performance is a good estimate of performance on unknown compounds that are drawn from the same distribution as the training set. The 'in catalog' metric is the performance when the model has seen the test example. The variance

61 in the second column of Table 2 to the give the "percent of variance explained". Table 3 shows examples of accurately predicted molecules from each price category and their predictions from 'in catalog' and OOB approaches. Table 4 shows molecules with the largest residual errors in the $\mathrm{OOB}$ predictions. These molecules are reference points to illustrate the molecules the model has the most trouble with.

\section{Analysis of model with 'model space' pairs}

To understand and improve the model, we inspected the reagents which produced the largest errors in the model predictions. We 

was the proximity distance of the random forest, as described by Brieman and Cutler[22]. In standard cliff pairs the distance is calculated from the fingerprints and thus has an 'unsupervised' character, whereas in our variant the trained model itself defines the distance. These cliff pairs allow us to inspect the model's pairwise discrepancy and see where the model failed to differentiate between two differently priced reagents ${ }^{18}$. Table 5 shows the results of these calculations for the molecules in Table 4 . Column 1 of Table 5 shows the reference molecule. Column 2 shows the three nearest neighbors in model space. Column 3 shows the catalog price of the molecule in column 1. Column 4 shows the catalog price of the neighbor. Column 5 shows the catalog number of the neighbor. Column 6 shows the Random Forest distance which is the number of trees that two molecules fall into different leaves. Table 6 shows pairs of molecules which are identical in our descriptor space, but which have different prices.

\section{Results and Discussion}

\section{Model performance}

Figures 1 and 2 and Table 1 show the model performance. Figure 1 shows the out-of-bag performance [20]. The OOB prediction performance of $34 \%$ variance demonstrates that the model is not so good at predicting new molecules that are similar those in the training set. Figure 2 shows a performance of $91 \%$ variance explained for reagents that the model has already seen ('in catalog').

Ordinarily, in machine learning, the prediction performance on the training set itself is a misleading metric for practical applications

of the model. However, in the usual case of ordering known reagents, the 'in catalog' performance is a metric of interest.

\section{Analysis of error}

The OOB prediction performance was disappointingly low. To put it another way: for this data set, as characterized by our descriptors, the Random Forest was not able to find generalizable patterns. To try to understand how the model is analyzing the data, we inspected some examples in which the model did poorly in estimating cost (Table 4).

To analyze the model we used our cliff pairs variant (see Methods) to identify the examples in each of the neighborhoods of the poorly predicted reagents. Table 5 shows the 3 nearest neighbors of each of the five poorly predicted compounds. For each of these five compounds, their nearest neighbors are very similar molecules which have very different prices. Given the similarity of the molecule and the difference in the price, it is not surprising that the OOB estimated price for this molecule is so far off.

Table 6 shows pairs of molecules (with different prices) that were indistinguishable by our descriptors and thus had identical is more expensive. 
For the typical use case of predicting on known reagents, our model (chiral Morgan fingerprints, RDKit medicinal chemistry descriptors, racemic/chiral center counts) performs at $91 \%$ variance explained. However, our model does not generalize well to unknowns, as reflected by its modest OOB performance of $34 \%$ variance explained. Thus, the model would need to be retrained as new compounds are added to the catalog.

Although beyond the scope of this work, we propose several avenues for model improvement for future investigation. Our first proposal is that an in-depth exploration into the difference economics of making each of the molecule pairs in Table 5 might provide insight into why such seemingly similar molecules have such different pricing and suggest performance enhancing descriptors.

104 For the molecules in Table 6, it is apparent that geometric isomers have different prices and thus an improved model would be able to distinguish them. In particular, the differences between cis-trans geometric isomers in ring structures, are not picked up by the chiral Morgan fingerprints. One way to capture this effect is to use descriptors that are sensitive to geometry. Pogliani[23] has suggested descriptors that can distinguish cis/trans geometry. Another possible descriptor would be an estimate of the strain in the molecules. tem.

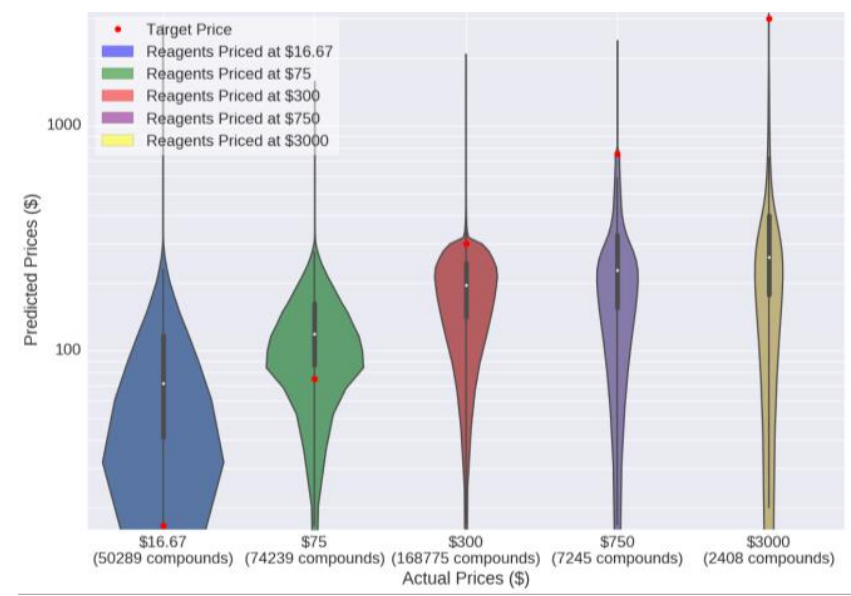

115 Each violin/segment in this plot shows the prediction for that price category. The overall performance for the model shown is $34 \%$ of variance explained.

\section{Figure 2. Violin Plot for Price Prediction of Known Reagents}




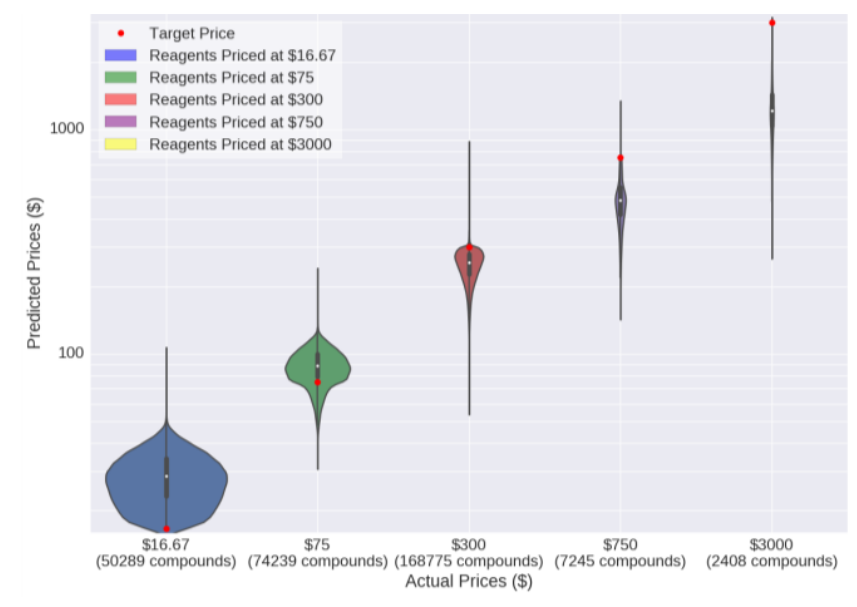

118 Each violin/segment in this plot shows the prediction for that price category. The overall performance for estimating a price that is in the catalog is $91 \%$ of 119 variance explained.

120

121 Table 1. Pricing Information

\begin{tabular}{|c|c|c|c|}
\hline $\begin{array}{c}\text { Price } \\
\text { category }\end{array}$ & $\begin{array}{c}\text { Numerical } \\
\text { value }\end{array}$ & $\begin{array}{c}\log _{10} \\
\text { (Numeri- } \\
\text { cal } \\
\text { value) }\end{array}$ & $\begin{array}{c}\text { Number } \\
\text { of exam- } \\
\text { ples in } \\
\text { data set }\end{array}$ \\
\hline$<50$ & 16.67 & 1.2 & 50289 \\
\hline $50-100$ & 75 & 1.875 & 74239 \\
\hline $100-500$ & 300 & 2.48 & 168775 \\
\hline $500-1000$ & 750 & 2.87 & 7245 \\
\hline$>1000$ & 3000 & 3.48 & 2408 \\
\hline
\end{tabular}

Table 2. Model Performance

\begin{tabular}{|c|c|c|c|}
\hline & $\begin{array}{c}\text { Variance } \\
\text { of the }\end{array}$ & $\frac{\text { Residual Variance }}{\text { Data Variace }}$ & (Variance \\
& Residual & Explained) \\
\hline $\log _{10}$ & & 0.66376 & \\
Price: & 0.16433 & & 0.33624 \\
OOB & & & \\
\hline
\end{tabular}




\begin{tabular}{|c|c|c|c|}
\hline Estimate & & & \\
\hline $\log _{10}$ & & & \\
Price: & \multirow{2}{*}{0.02232} & 0.09015 & \\
Known & & & 0.90985 \\
Reagents & & & \\
\hline
\end{tabular}

125

126

Table 3. Accurately Predicted Reagents

127

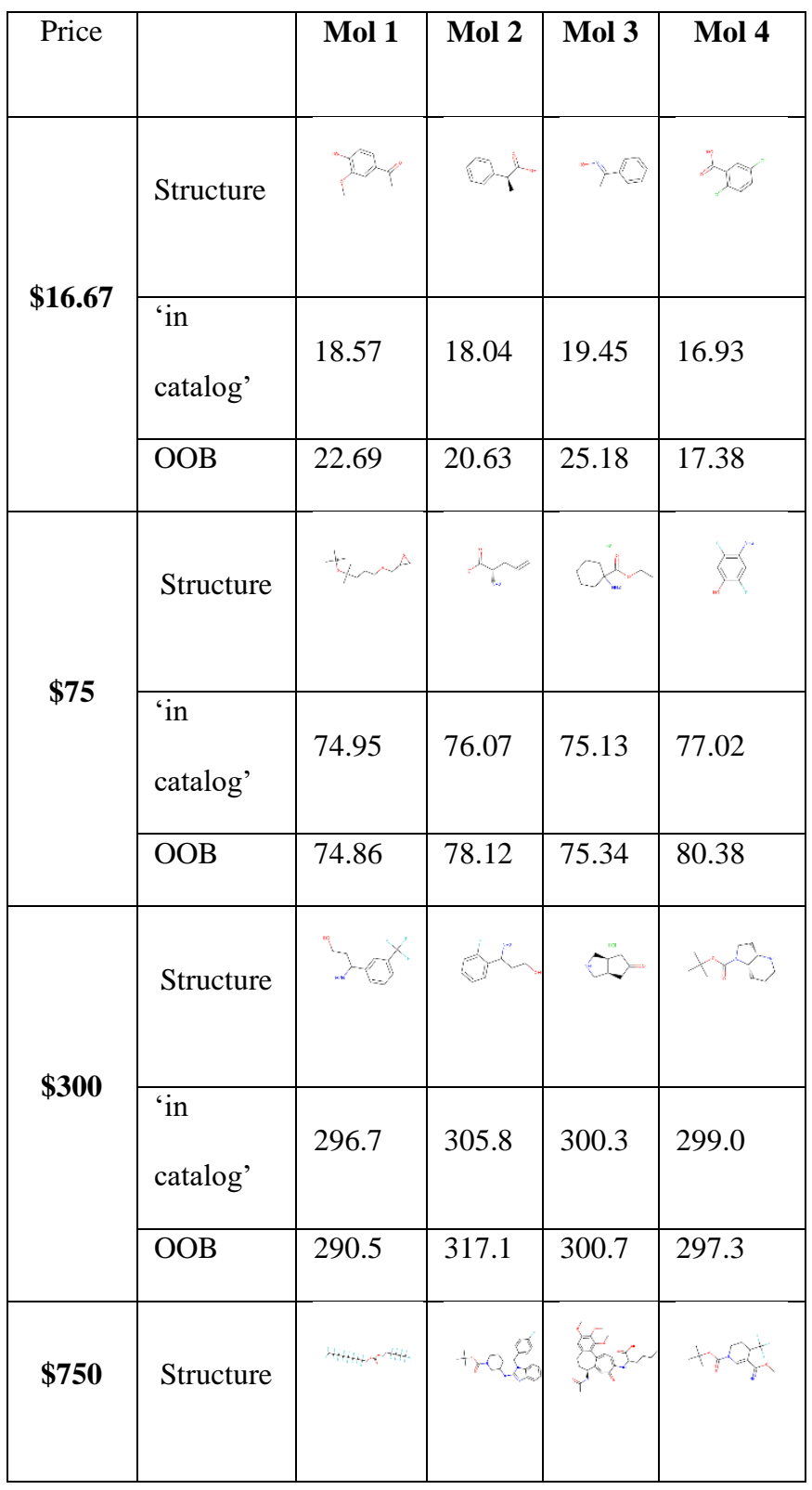




\begin{tabular}{|c|c|c|c|c|c|}
\hline & $\begin{array}{l}\text { 'in } \\
\text { catalog' }\end{array}$ & 750.0 & 752.5 & 751.6 & 750.2 \\
\hline & $\overline{\mathrm{OOB}}$ & 750.0 & 757.0 & 754.8 & 750.6 \\
\hline \multirow{3}{*}{$\$ 3000$} & Structure & & $a r$ & 208 & 328 \\
\hline & $\begin{array}{l}\text { 'in } \\
\text { catalog' }\end{array}$ & 2995.8 & $\begin{array}{l}2988 . \\
7\end{array}$ & $\begin{array}{l}2980 . \\
7\end{array}$ & 2973.82 \\
\hline & OOB & 2993.1 & $\begin{array}{l}2981 . \\
9\end{array}$ & $\begin{array}{l}2949 . \\
15\end{array}$ & 2927.45 \\
\hline
\end{tabular}

129 


\begin{tabular}{|c|c|c|c|c|}
\hline & Structure & $\begin{array}{c}\text { OOB } \\
\text { Predic- } \\
\text { tion }\end{array}$ & Price & $\begin{array}{c}\text { MolPort } \\
\text { Id }\end{array}$ \\
\hline 1 & & 2735 & 16.67 & $\begin{array}{c}\text { Mol- } \\
\text { Port-001- } \\
\text { 785-612 }\end{array}$ \\
\hline 2 & & 36.03 & 3000 & $\begin{array}{c}\text { Mol- } \\
\text { Port-003- } \\
\text { 927-365 }\end{array}$ \\
\hline 3 & & 30.25 & 3000 & $\begin{array}{c}\text { Mol- } \\
\text { Port-001- } \\
\text { 792-474 }\end{array}$ \\
\hline 4 & $\begin{array}{l}30 \\
3030 \\
30\end{array}$ & 45.47 & 3000 & $\begin{array}{c}\text { Mol- } \\
\text { Port-042- } \\
\text { 652-610 }\end{array}$ \\
\hline 5 & & 30.79 & 3000 & $\begin{array}{c}\text { Mol- } \\
\text { Port-003- } \\
939-463\end{array}$ \\
\hline
\end{tabular}

131

132

133 Table 5. Reagents with the Largest Residual Errors and their Cliff Pairs

\begin{tabular}{|l|l|l|l|l|l|l|}
\hline Mol 1 & Mol 2 & Price 1 & Price 2 & MolPort & RF Dist \\
\hline 1 & & & & & MolPort- & 566 \\
\hline & & & & & & \\
\hline
\end{tabular}




\begin{tabular}{|c|c|c|c|c|c|c|}
\hline & & $\frac{2}{2}$ & & 3000 & $\begin{array}{l}\text { MolPort- } \\
\text { 030-042- } \\
387\end{array}$ & 627 \\
\hline & & 3 & & 3000 & $\begin{array}{l}\text { MolPort- } \\
\text { 030-042- } \\
404\end{array}$ & 640 \\
\hline & & & & 16.67 & $\begin{array}{l}\text { MolPort- } \\
\text { 001-779- } \\
706\end{array}$ & 568 \\
\hline 2 & & & 3000 & 16.67 & $\begin{array}{l}\text { MolPort- } \\
001-779- \\
902\end{array}$ & 581 \\
\hline & & & & 16.67 & $\begin{array}{l}\text { MolPort- } \\
\text { 001-770- } \\
158\end{array}$ & 685 \\
\hline \multirow{3}{*}{3} & \multirow{3}{*}{${ }^{2} \gamma_{\mathrm{s}}$} & & \multirow{3}{*}{3000} & 16.67 & $\begin{array}{l}\text { MolPort- } \\
\text { 001-792- } \\
487\end{array}$ & 540 \\
\hline & & $x$ & & 16.67 & $\begin{array}{l}\text { MolPort- } \\
\text { 023-278- } \\
197\end{array}$ & 713 \\
\hline & & 23 & & 16.67 & $\begin{array}{l}\text { MolPort- } \\
\text { 006-121- } \\
512\end{array}$ & 721 \\
\hline 4 & $2000^{800}$ & $80^{808}$ & 3000 & 16.67 & $\begin{array}{l}\text { MolPort- } \\
\text { 005-937- } \\
160\end{array}$ & 647 \\
\hline
\end{tabular}




\begin{tabular}{|c|c|c|c|c|c|c|}
\hline & & & & 16.67 & $\begin{array}{l}\text { MolPort- } \\
\text { 019-903- } \\
928\end{array}$ & 709 \\
\hline & & 808080 & & 16.67 & $\begin{array}{l}\text { MolPort- } \\
\text { 023-223- } \\
115\end{array}$ & 756 \\
\hline \multirow{3}{*}{5} & \multirow{3}{*}{$r a$} & & \multirow{3}{*}{3000} & 16.67 & $\begin{array}{l}\text { MolPort- } \\
\text { 023-282- } \\
744\end{array}$ & 628 \\
\hline & & 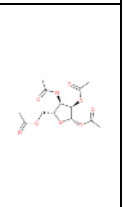 & & 16.67 & $\begin{array}{l}\text { MolPort- } \\
\text { 000-158- } \\
507\end{array}$ & 674 \\
\hline & & & & 16.67 & $\begin{array}{l}\text { MolPort- } \\
\text { 003-871- } \\
476\end{array}$ & 818 \\
\hline
\end{tabular}

134

135

136 Table 6. Reagents with Identical Model Space, but with Different Prices

\begin{tabular}{|l|c|c|c|c|}
\hline & Mol 1 & Mol 2 & Price 1 & Price 2 \\
\hline $\mathbf{2}$ & $\mathbf{2}$ & & & \\
\hline & & & & \\
\hline
\end{tabular}




\begin{tabular}{|c|c|c|c|c|}
\hline 3 & $\underbrace{+}$ & and & 300 & 16.67 \\
\hline 4 & 索 & 声 & 3000 & 300 \\
\hline 5 & 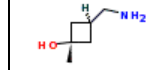 & 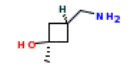 & 750 & 300 \\
\hline
\end{tabular}

138

139

140

141

142

143 Supplemental Table 1. Reagents with Identical Model Space, but with Different Prices

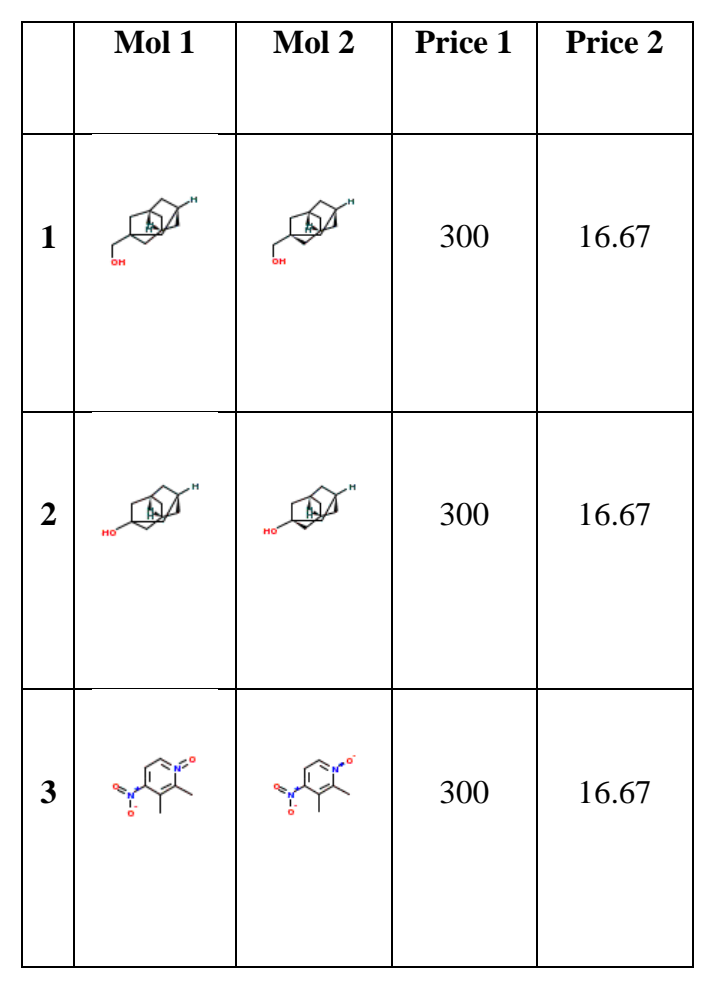

144

145 DECLARATIONS 


\section{Availability of data and materials}

147 The data file from MolPort and an e-mail giving us permission to use it are available in the supplementary section. RDKit, sci-kit 148 are open source software.

\section{Competing interests}

150 The authors work was funded of Novartis Institute for BioMedical Research.

\section{$151 \quad$ Funding}

152 Kwabena Ofori-Atta was a D\&I summer scholar and then intern in the Global Discovery Chemistry. Clayton Springer is an em153 ployee of Novartis

\section{Authors' contribution}

156 CS proposed the project and supervised the work. KOA did the work and wrote the first draft of the manuscript.

\section{Acknowledgements}

158 We thank Molport for allowing us to share their catalog information.

\section{Authors' information}

160 Kwabena Ofori-Atta (kobbie@ mit.edu)

161 Clayton Springer (clayton.springer@ novartis.com) 


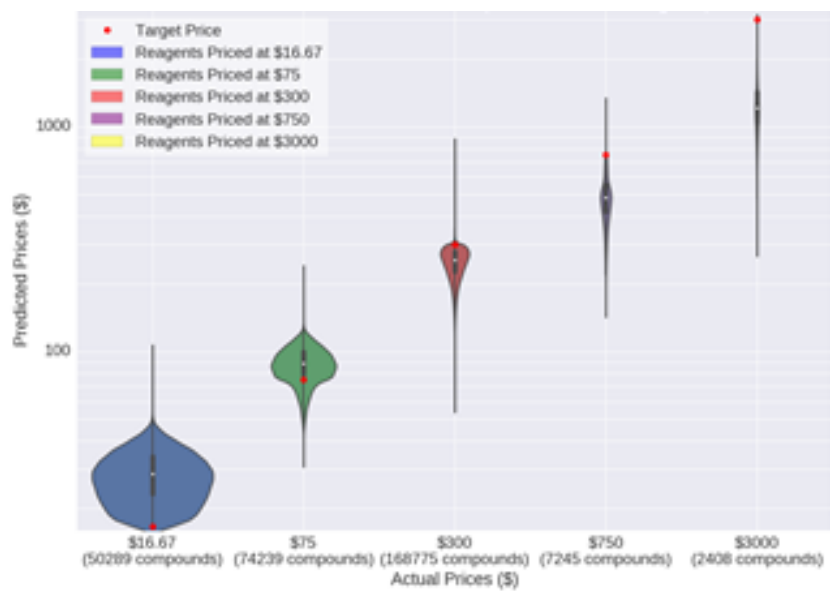
multiobjective genetic algorithm. J Chem Inf Comput Sci. 2002;42(2):375-85.

171 2. Dolle RE. Historical overview of chemical library design. Methods Mol Biol. 2011;685:3-25.

172 3. Beno BR, Mason JS. The design of combinatorial libraries using properties and 3D

173 pharmacophore fingerprints. Drug Discov Today. 2001;6(5):251-8.

174 4. Blaney JM, Martin EJ. Computational approaches for combinatorial library design and 175 molecular diversity analysis. Curr Opin Chem Biol. 1997;1(1):54-9.

176 5. Chen H, Börjesson U, Engkvist O, Kogej T, Svensson MA, Blomberg N, et al. ProSAR: A New 177 Methodology for Combinatorial Library Design. Journal of Chemical Information and Modeling. $178 \quad 2009 ; 49(3): 603-14$.

179 6. Ebalunode JO, Zheng W, Tropsha A. Application of QSAR and shape pharmacophore modeling 180 approaches for targeted chemical library design. Methods Mol Biol. 2011;685:111-33. 
181 7. Gillet VJ. Reactant- and product-based approaches to the design of combinatorial libraries. Mol 182 Divers. 2002;5(4):245-54.

183 8. Gillet VJ. Designing combinatorial libraries optimized on multiple objectives. Methods Mol 184 Biol. 2004;275:335-54.

185 9. Gregori-Puigjane E, Mestres J. Coverage and bias in chemical library design. Curr Opin Chem 186 Biol. 2008;12(3):359-65.

187 10. Leach AR, Hann MM. The in silico world of virtual libraries. Drug Discov Today. $188 \quad 2000 ; 5(8): 326-36$.

189 11. Willett P. Chemoinformatics - similarity and diversity in chemical libraries. Curr Opin 190 Biotechnol. 2000;11(1):85-8.

191 12. Yasri A, Berthelot D, Gijsen H, Thielemans T, Marichal P, Engels M, et al. REALISIS: a 192 medicinal chemistry-oriented reagent selection, library design, and profiling platform. J Chem Inf 193 Comput Sci. 2004;44(6):2199-206.

194 13. Zheng W, Cho SJ, Tropsha A. Rational combinatorial library design. 1. Focus-2D: a new 195 approach to the design of targeted combinatorial chemical libraries. J Chem Inf Comput Sci. $196 \quad 1998 ; 38(2): 251-8$.

197 14. Good AC, Lewis RA. New methodology for profiling combinatorial libraries and screening sets: 198 cleaning up the design process with HARPick. J Med Chem. 1997;40(24):3926-36.

199 15. Kalliokoski T. Price-Focused Analysis of Commercially Available Building Blocks for 200 Combinatorial Library Synthesis. ACS Combinatorial Science. 2015;17(10):600-7.

201 16. Ertl P, Schuffenhauer A. Estimation of synthetic accessibility score of drug-like molecules based 202 on molecular complexity and fragment contributions. J Cheminform. 2009;1(1):8.

203 17. Fernandez M, Ban F, Woo G, Isaev O, Perez C, Fokin V, et al. Quantitative Structure-Price 204 Relationship (QS\$R) Modeling and the Development of Economically Feasible Drug Discovery 205 Projects. Journal of Chemical Information and Modeling. 2019. 
206 18. RDKit. RDKit: Open-source cheminformatics. 2018.

207 19. Fabian Pedregosa, Gaël Varoquaux, Alexandre Gramfort, Vincent Michel, Bertrand Thirion, 208 Olivier Grisel, et al. Scikit-learn: Machine Learning in Python. Journal of Machine Learning Research. $209 \quad 2011 ; 12: 2825--30$.

$21020 . \quad$ Breiman L. Random Forest. 2001.

211 21. Guha R, Van Drie JH. Structure--activity landscape index: identifying and quantifying activity 212 cliffs. J Chem Inf Model. 2008;48(3):646-58.

213 22. Breiman L, Cutler A. Random Forests proximity. 2001.

214 23. Pogliani L. On a Graph Theoretical Characterization of Cis/Trans Isomers. J Chem Inf Comput 215 Sci. 1994;34:801-4.

216 